\title{
Clinical Trials Legislation - Preparing for the Revision of the European Directive Scheduled for 2011
}

\author{
Pascal Bilbault ${ }^{1}$, Chantal Belorgey ${ }^{2}$, Faiez Zannad ${ }^{3}$, Danielle Golinelli ${ }^{4}$, Yannick Pletan ${ }^{5}$ and participants \\ of Round Table $n^{\circ} 4$ of Giens XXV ${ }^{\star}$ \\ 1 Laboratoire Boehringer Ingelheim, Reims, France \\ 2 Afssaps, Saint-Denis, France \\ 3 Centre d'Investigation Clinique, Hôpital Jeanne d'Arc, Dommartin les Toul, France \\ 4 Direction Générale de la Santé, Paris, France \\ 5 Laboratoire Pfizer, Paris, France
}

Text received 29 march 2010; accepted 23 april 2010

\section{Keywords:}

clinical trials; european Directive; biomedical research; revision; risk

\begin{abstract}
The aim of the Round Table was to make recommendations with regard to the imminent revision of the European Directive on clinical drug trials (2001/20/CE).

While recognising the importance of compliance with this Directive, which is not optimal in some member states of the European Union, it would be constructive to simplify further and harmonise its application in every country.

Without necessarily resorting to a revision, some of the Directive's dispositions could be improved, such as the definition of "investigational medicinal products" (IMP) and what should be considered as "substantial amendements", as well as harmonising and improving the way in which Ethics Committees are run, either on a European Commission level, or by relying more on the European Network of Research Ethics Committees (EUREC) which already exists in several European member states.

Other points in the Directive do require revision, especially those relating to:

- the definition of the respective roles of Ethics Committees and Competent Authorities,

- the simplification of safety information to Ethics Committees (giving them access to the Eudravigilance database for adverse reactions occurring during clinical trials and providing them with only new safety issues or with a summary of the Annual Safety Report),

- the possibility of one single European authorisation for the trial, centralised and/or decentralised, when the trial is multinational.

The recommended changes could be made within the scope of a European Regulation, avoiding the need to transpose it at a later date into each country's regulations, which is a source of possible lack of harmonisation. More specifically, for trials with institutional sponsors and/or investigator driven trials such as "drug therapy strategy trials", modulating restrictions according to the "risk added by the research" would enable the trial's organisation to be simplified regarding monitoring, adverse reactions reporting and study drugs labelling.
\end{abstract}

\section{Introduction}

Since its application, the European Directive 2001/20/CE on clinical trials on medicinal products has led to regulations between the different countries of the European Union being harmonised,

\footnotetext{
* For a list of participants, see end of article.
}

especially with regard to the application of good clinical practices, patient protection (especially vulnerable patients), modalities for conducting clinical trials (the prior authorisation system and the quality of IMPs) and information sharing between national Competent Authorities (NCA) of member states with the creation of the EudraCT database. 
Table I. Opinions of european investigators, authors of trials, on the EC that assessed them.

\begin{tabular}{lc}
\hline 5 questions put to investigators on their interaction with EC & Average score* \\
\hline - The EC provided sound support & $-2,5$ \\
- The effort required to obtain authorisation encouraged us to make progress with regard to ethics & $-3,7$ \\
- The effort required to obtain authorisation encouraged us to make scientific progress & $-4,1$ \\
- The effort required to obtain authorisation was justified & $-1,1$ \\
- Authorisation was granted in a courteous atmosphere & 2,0 \\
\hline
\end{tabular}

- score between -10 (extremely unsatisfactory) and +10 (extremely satisfactory)

EC: Ethic Committees

However, for the sponsors of clinical trials, both institutional and industrial, this major progress comes with administrative complexities and a significant increase in workload as well as divergences in interpretation between member states.

Therefore, during the debate on the need to revise the European Directive (scheduled for 2011), it is important to make recommendations on a French level.

Specifically, we considered the viewpoint of institutional and industrial stakeholders as well as that of Comités de Protection des Personnes ["CPP" research Ethics Committees (EC)], and concentrated our debate on 3 predefined points:

1. How to contribute so that the current Directive is better applied in certain European member states?

2. How to simplify it further?

3. How to harmonise its application in all European countries?

Furthermore, a chapter is devoted to a proposal for a new approach based on the risk added by research.

Lastly, we offer a summary of desirable recommendations on a European level.

\section{Institutional sponsors' viewpoint}

A recent study ${ }^{[1]}$ illustrates the point of view of European investigators who have authored clinical trials published in major medical journals with regard to the European EC who had given opinions on these studies (table I). Investigators were mostly dissatisfied with both the content (the Committees' expertise and competence) and the form (submission procedures and the quality of the contact).

On a European level, institutional sponsors have been taking several initiatives for some years, notably at the instigation of investigator networks such as European Clinical Research Infrastructures Network (ECRIN) or through the Impact on Clinical Research of European Legislation (ICREL project) ${ }^{[2]}$ or groups of research bodies such as the European Science Foundation (ESF).
We could, for example, draw attention to ECRIN's ${ }^{[3]}$ survey which confirmed the very great heterogeneity of national regulations (especially those concerning research which does not involve medicinal products). The ICREL's study also confirms that the total number of drug trials did not drop after the Directive was established, but that the time spent setting them up has increased by $24 \%$ in 3 years, with a notable increase in the workload. ${ }^{\text {[2] }}$

These initiatives all come to the conclusion we need to simplify procedures to assess clinical trials on medicinal products in Europe: the assessments could be made by a single CA in line with the Voluntary Harmonisation Procedure (VHP) for clinical trials, established on the Clinical Trial Facilitation Group (CTFG) initiative. We would also like to have harmonised insurance systems, and lastly, would like to agree on a typology of trials based on the level of risk linked to the research. The way the various ECs function, which is not yet perfect, and their lack of harmonisation in Europe and the need, if possible, for a single, certified EC per country are also recurring themes.

The 2001/20/CE European Directive, however, provides for a protocol to be examined by a single EC per country. This is still not applied in every member state, especially those which are structured by region (Germany, Spain). It has not been proved that the multiplicity of opinions has improved projects' ethical and scientific aspects.

As far as France is concerned, it also appears to have become more difficult for institutional sponsors to organise clinical trials than for industrial sponsors since the European Directive was applied in France, despite information available on a regulatory level.

The Jardé Bill ${ }^{[4]}$ currently under discussion should facilitate some institutionally promoted trials by creating for non drug trials an additional category of interventional research with negligible risk compared with normal use (broadening the existing notion of research aiming to assess usual care). With regard to drugs, phase IV therapeutic strategy trials which are of interest for numerous academic networks, remain, however, biomedical research from a legal point of view and are subject to the European Directive. This framework is not necessarily adapted due to administrative 
and regulatory obligations which consume a large amount of resources, and, in the future, institutional sponsors are in danger of turning away from this type of study. A new approach based on the risk added by research could reduce obligations for trials in terms of procedures to assess, monitor and manage adverse reactions.

\section{Ethics Committees (CPP) viewpoint}

The role of EC varies from one European country to another. While the respective roles of EC and of the CA are clearly defined by legislation in France, this is not the case everywhere in Europe.

The CTFG carried out a study in 2008 into the respective roles and responsibilities of CA and EC in most European countries. ${ }^{[5]}$ Patient information and informed consent forms were analysed by both organisations in half the countries involved. Drugs files are generally assessed by the CA, but 4 countries also confer this task to the EC for part of the file. Method assessment is conferred to $\mathrm{EC}$ in 4 countries, to the $\mathrm{CA}$ in 3 and divided between EC and CA in the other countries. Submission occurs at the same time to EC and CA in the majority of countries, but the modalities of opinions vary from the single opinion of an EC for all centres such as in France, Portugal or Greece, to more complex systems. We thus have a different procedure depending on whether the study is monocentric with an opinion from the local $\mathrm{EC}$, or multicentric with an opinion from the central EC. This is the case, for example, in Austria or Finland. The situation of countries such as Germany or Italy is also very complex, with opinions from both local and central EC. Some aspects of the file are dealt with by one of the official organisations and others by the other organisation.

In the end, although the Directive demands a single opinion, the procedures for issuing this opinion are sometimes complex and may call upon one or several organisations, depending on the country.

But what exchanges take place between the two organisations, both during analysis of the file and in passing on opinions from one organisation to the other?

A recent study into this matter was carried out by one of the members of the round table among his national correspondents and 19 countries responded.

In the majority of cases, there is no exchange between the EC and the CA during analysis of the file. However, most EC pass on their opinion to the CA, with Poland the exception to the rule. In the other direction (CA to EC) in France, the CA's comments are automatically passed on to the CPP concerned. Other national CA do not pass on their remarks to EC. Similarly, the CA's ultimate decision is passed on to the EC in only 4 countries out of 20 .
Spain is a unique case, with a website allowing constant exchanges between EC and the CA, both during the process of examining the file and for the definitive decision.

France differs from other countries in that it transmits unfavourable opinions from each CPP to all the other CPP. In the event of an unfavourable opinion from the EC, some countries including France have established a procedure for a second examination by another EC.

Another potential area for improvement is the composition of EC. The Directive stipulates that the Committee be composed of an equal number of members from civil society and scientific experts, but this is not applied in every country. Each country is free to decide the number of members, how they are nominated and which qualifications are required. Depending on the country, Committees may have from 7 to 25 members.

\section{1. EC (CPP) recommendations}

Having completed our analysis, it appears to us that one of the main recommendations for better harmonisation would be for one single EC per member state to examine an identical file for all countries.

It is hard to imagine a unique European ethical opinion for a clinical trial for all countries. Of course, an approach based on general principles of the ethical nature of the research with regard to scientific knowledge in the area involved would be conceivable. However, it is vital to maintain an ethical opinion per country, as this is the only approach which can take into account each country's cultural specificities and how the opinion given for information and informed consent forms is expressed.

Smoother functioning and improved harmonisation between EC could also be obtained as follows:

- Intra country: harmonised files, unique format, single information point, specimen opinion, guidelines.

- Inter country: harmonised files, unique format, better harmonisation of the composition of $\mathrm{EC}$ and of exchanges between $\mathrm{EC}$ and $\mathrm{CA}$.

- Electronic tool (inter EC communication, access to EudraCT).

- Training and certification.

Another recommendation would be to make more use of the EUREC, ${ }^{[6]}$ a network of EC which already includes a dozen European countries, with a view to harmonising practices and decisions.

\section{Industrial sponsors' viewpoint}

The observation on an industrial level concurs with the above mentioned observations: the Directive has indisputably made a 
significant contribution in Europe, and commercial sponsors have organised themselves accordingly to set up and manage their clinical trials. In the CT safety domain, the Directive has meant that the same language is generally being spoken, and, in theory, it offers better protection for patients by exchanging more information relating to safety between the parties involved.

However, some of the Directive's harmonisation disappeared once it was transposed by member states who introduced differences into its scope of application, in authorisation times ("clockstop" during assessment time in some countries), in requests for additional documentation, in the definitions of investigational medicinal products and substantial amendements, and lastly, in reporting adverse reactions by introducing numerous national specificities. A survey of pharmaceutical industry parent companies or European Head Offices has confirmed difficulties linked to different interpretations depending on the country, to diverging opinions between national CA or between national CA and EC and to the heterogeneity of EC resulting in local Committees making more scientific demands than national Committees, for example.

Generally speaking, the workload has increased for CA and $\mathrm{EC}$ as well as for sponsors. This increase is due to additional regulatory requirements to set up the Directive, but also with regard to the need for sponsors to comply with every national specificity. At the same time, this increase in activity has led to increased costs, in particular due to the need for additional resources.

ECs are encountering operational problems as the manual management of all the paper documents becomes increasingly complex, notably for safety data with real constraints for reviewing and analysing submitted documents, especially all the individual adverse reactions observations. Suspected unexpected serious adverse reactions (SUSARs) are passed on by sponsors without being analysed and are sent to $\mathrm{CA}$ at the same time. Moreover, this large quantity of "raw" information does not allow the most relevant elements to be highlighted, which would be more appropriate for the protection of trial subjects.

ECs also receive SUSAR line listings, generally sent without any comment and at a rhythm which varies from one country to another (usually twice-yearly, but sometimes every 3 months). As for Annual Safety Reports (documents which are more and more voluminous and which require no formal assessment) in general, they are rarely reviewed either by ECs or by CA.

This observation is shared by investigators who, for every trial carried out on-site, receive too many individual serious cases, and who do not have time to familiarise themselves with all the information.

Altogether, a huge amount of safety data circulates, but the format and periodicity vary depending on the member state and often appear to be ill adapted to the missions and requirements of the parties involved. In consequence, this calls into question the relevance of supplying such data to protect subjects who participate in research, all the more so as, generally, EC have neither the expertise nor the resources, nor the organisation to manage this mass of information.

\subsection{Commercial sponsors' recommendations}

Pending a revision of the European legislation, an initial set of improvements could be suggested without having to modify the Directive's original wording.

- This would involve further harmonising the application of the Directive in Europe by drawing up common definitions and additional explicative guidelines: the definition of investigational medicinal products, substantial amendments and the type of research (observational or interventional).

- Recommendations on the content of the clinical trial subject information and consent are also advisable, as well as on filling in EudraCT request forms. These definitions and recommendations could be drawn up and validated in common by member states representatives at the CTFG level in order to be accepted in every country.

- It is necessary to define clearly the content of the files submitted to CA and those addressed to EC so that they are not subject to additional national requests and are sufficiently precise to avoid diverging local interpretation.

- A clear allocation of the respective responsibilities of the two authorities (Competent and Ethics) in terms of assessment would avoid contradictory requests. Lastly, a common European single point to submit request files is strongly advisable and the use of electronic means should be developed in order to better communicate with ECs.

- In the short term, it would be extremely useful to apply this harmonisation and simplification in France. Two proposals which were discussed during the round table could quickly be the subject of consensus. One is a "template of the CPP opinion" which would enable all the compulsory information to be found as set out in the modified decree dated May $24^{\text {th }}$ 2006. Furthermore, the different demands from the various CPP should not be acceptable because a decree already stipulates in France what should be in the CPP file for a clinical drug trial. Common submission modalities could be proposed (number of copies, submission schedule).

- For safety, it would be useful to simplify obligations by reducing unnecessary reporting and avoiding the duplication of activity relating to CA. The modalities for declaring SUSARs should also be identical with regard to the definition in every member state. 
A second level of improvements would require text modifications at the European level (changes in the Directive):

- Significant changes could be proposed, such as a European authorisation for biomedical research along the lines of European marketing authorisation procedures (centralised or decentralised procedure). This new procedure would be an alternative to the national procedure currently in force. Ultimately, the type of procedure chosen should remain the choice of the sponsor. This alternative would remove several obstacles currently encountered by sponsors in terms of time, administrative complexity and additional costs.

- Moreover, significant changes could also be made to CT safety management to maintain EC's access to all individual safety data, without overloading them with irrelevant systematic information. It has been suggested to give ECs access to Eudravigilance CT module and thus to abolish the regular sending out of SUSARs as well as twice-yearly lists. It should be borne in mind that only CA have the power to carry out health inspections with regard to safety information. Regarding the Annual Safety Report (ASR) which is undergoing changes in favour of the Development Safety Update Report (DSUR), only the report's summary would be sent to the ECs once a year.

\section{Proposal for a new approach based on added risk from research}

It now seems necessary to try to define a new approach based on risk added by the research compared to usual care. Although the topic has a European impact and certain ECs in countries such as Austria have started to move into this direction, for the moment we are concentrating on research cases scheduled in France.

Three types of possible consequences exist for an approach (which could be made more flexible), based on the risk added by the research:

- Consequences for the safety of patients taking part in the study.

- Consequences for the quality of data:

- because of the possible impact on a Marketing Authorisation file if research involves a drug (or medical device),

- because of the possible impact of research results through publication or consensus conference, on the change of treatment (diagnostic and/or therapeutic) for a given pathology.

- Advertising, brand-image or financial consequences.

The only 2 first approaches (patient safety, quality of data) were considered by the round table.
Several models for risk added by research regarding patient safety have already been suggested, notably in France:

- An initial 3-level model was designed at the Giens pharmacology workshop in 2005 in view to adapting monitoring. ${ }^{[7]}$

- The AP-HP (Paris area's public hospital system) 4-level model, established to adapt the monitoring on the risk to patients added by research. This model has been approved by the Afssaps (French Medicines Agency) and is used by other sponsors.

- A risk level corresponds to the lowest risk (i.e. blood sample or non-invasive examination),

- D risk level corresponds to the highest risk (new drugs or medical device trials, gene therapy, cell therapy). This is associated with monitoring of $100 \%$ of files (of important data),

- $\mathrm{B}$ and $\mathrm{C}$ risk levels are intermediate.

For a clinical trial on medicinal product, the normal risk level should be $\mathrm{C}$ or $\mathrm{D}$, and can only be $\mathrm{B}$ if it involves a trial assessing a drug which already has a Marketing Authorisation and is used in the approved conditions. The risk level also directly influences the decision whether or not to set up a Data Safety Monitoring Board (DSMB), which should be systematic in the event of D Risk, debatable in the event of C Risk and unnecessary in the event of A or B Risk. Exemptions for the free supply of drugs by institutional sponsors are provided by the article L-1121-16-1 of the French Health Code, especially when the trial involves one or more pharmacological classes (the choice of which is left to the investigator's appreciation but which remains within the scope of an indication agreed by the Marketing Authorisation). In those situations, Afssaps may accept specific modalities of traceability for trial treatments.

- A simplification of the AP-HP model to 3 levels was drawn up in 2009 by an AP-HP \& Inserm working group and is currently under discussion by the Institutional Sponsor Organisation (CPI, Coordination des Promoteurs Institutionnels).

Although no priori tool exists to determine risk before full analysis of the documentation by the CA or CPP, some trials could be subject to a self-assessment of risk level by the sponsor depending on criteria to be defined. The sponsor would thus adapt its monitoring and resources according to the expected risk level for each biomedical research project:

1. Depending on the level of added risk from research for patients, as declared to the authorities and insurance company. The suggested minimum monitoring must guarantee respect for the patient's protection and rights as well as his/her safety: information and consent, deviations from protocol, dispensation of treatment and serious adverse reactions. Monitoring 
must allow for the possibility to amend the protocol in order to better protect the clinical trial subject (changes of eligibility criteria, follow-up and possibly treatment dosage) and also allow for the eventuality of information being supplied to a DSMB who would then be responsible for making recommendations (or even demand a premature trial discontinuation).

2. Depending on the desired level of data quality control (whether or not the trial is risky for the patient) depending on certain variables (trial for a registration file or when data are bought by a manufacturer; major impact on medical treatment). In practice, this is a question of knowing whether or not one wants to verify the quality of important data for $100 \%$ of patients.

In practice, it is possible to have a risk for patients assessed "minor" as declared to the authorities, but which is $100 \%$ monitored because the data are to be forwarded to a manufacturer or because it may have an international impact or modify patients' treatment (if one wishes to be certain of the veracity and reliability of the data and conclusions).

For trials of drugs with Marketing Authorisation for that therapeutic indication ("drug therapy strategy" type trials), institutional sponsors could simplify part of the organisation.

In all cases, the following conditions must be fulfilled:

- Good Clinical Practice (GCP) must be respected, whatever the level of monitoring.

- Sponsors' responsibility must remain the same (consent, declaration of serious adverse reactions).

In addition, it remains to be decided who should validate the risk level suggested by the sponsor (should this not be the CA rather than the EC?) as well as insurance and compensation conditions (the cost of insurance can indeed vary depending on the risk level announced for each study).

This subject is very topical and it would be worth analysing all the European experiences conducted in France (Optimon project), ${ }^{[8]}$ in the UK (risk approach depending on the trial suggested in the MRC toolkit), ${ }^{[9]}$ in Austria (Vienna's Ethics Committee) ${ }^{[10]}$ and in Germany (Adamon Project). ${ }^{[11]}$

The round table has listed all the items which could be adapted in a risk-based approach (table II).

\section{Summary of recommendations}

It is essential to bear in mind that the Directive's numerous benefits should be retained: authorisation time-frames, the possibility of national trial authorisation procedures, safety modalities and lastly the principles of subjects protection and GCP regulations. The main recommendations, without pre-judging the means
Table II. Non-exhaustive list of processes which could be adapted according to an approach based on risk added by research.

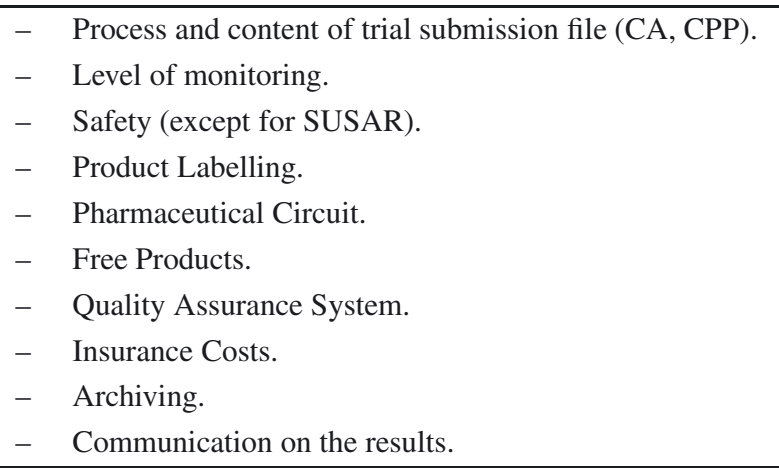

CA: Competent Authorities; CPP: Comités de Protection des Personnes; SUSAR: Suspected unexpected serious adverse reactions

of putting them into practice (changes of recommendations or of the wording of the Directive), are set out in table III.

In practice, in addition to the risk-based approach described above, recommendations on two levels have been suggested.

\section{1. Improving the application of the Directive}

A great number of these recommendations can be applied without changing the Directive, merely the Guidances (e.g. the definition of investigational medicinal products or substantial amendments or improving the running of EC and the harmonisation between them). On the other hand, it is essential to make sure that the Directive and its guidelines are applied in every country (the European Commission should verify its implementation). Finally, with regard to EC, the coordinating role of the EUREC should be clarified and strengthened, as is the case for the CTFG for matters concerning CA.

\subsection{Proposing changes for the Directive}

It would seem, then, that several of the Directive's items require significant changes, either by revising the current one or by adopting another legislative measure such as a European Regulation.

This, especially, is the case for defining the respective roles of $\mathrm{EC}$ and $\mathrm{CA}$ or for the nature of safety information to be passed on to EC. The members of the round table were unanimous regarding the latter point. It would mean giving EC access to Eudravigilance (CT module), the European database of adverse reactions occurring during the trial, which would enable the documentation received by EC to be reduced. The systematic reporting of all SUSARs and twice-yearly line listings would be abolished, 
Table III. Round Table's main recommendations.

\begin{tabular}{lll}
\hline Institutional sponsors & CPP & Industrial sponsors \\
\hline $\begin{array}{l}\text { Possibility of a single } \\
\text { and a single EC opinion } \\
\text { per country (with } \\
\text { certification of EC) }\end{array}$ & $\begin{array}{l}\text { Guidance on files for EC } \\
\text { applied in every country }\end{array}$ & $\begin{array}{l}\text { Better definition of } \\
\text { investigational medicinal } \\
\text { products and substantial } \\
\text { amendments }\end{array}$ \\
\hline $\begin{array}{l}\text { Modulation depending } \\
\text { on the risk added } \\
\text { by the research }\end{array}$ & $\begin{array}{l}\text { Harmonising the running } \\
\text { of EC on a European } \\
\text { Commission level (EUREC } \\
\text { or another identified group) }\end{array}$ & $\begin{array}{l}\text { Single European information } \\
\text { point for EC (electronic tool) }\end{array}$ \\
\hline $\begin{array}{l}\text { Harmonised insurance } \\
\text { system }\end{array}$ & $\begin{array}{l}\text { One single EC per country } \\
\text { or per region }\end{array}$ & $\begin{array}{l}\text { Only sending EC summaries } \\
\text { of ASR and new safety issues } \\
\text { rather than SUSARs and } \\
\text { twice-yearly line listings }\end{array}$ \\
\hline $\begin{array}{l}\text { Simplifying safety } \\
\text { obligations }\end{array}$ & $\begin{array}{l}\text { Better definition of the } \\
\text { respective roles and } \\
\text { responsibilities of CA and EC }\end{array}$ & $\begin{array}{l}\text { Better definition of the content } \\
\text { of respective CA and EC files }\end{array}$ \\
\hline & $\begin{array}{l}\text { Clarifying exchanges } \\
\text { between CA and EC }\end{array}$ & $\begin{array}{l}\text { Giving EC access to } \\
\text { Eudravigilance (CT module) }\end{array}$ \\
\hline & $\begin{array}{l}\text { Harmonising tasks and } \\
\text { composition of EC in every } \\
\text { member state }\end{array}$ & $\begin{array}{l}\text { Recommendations on content } \\
\text { of consent and EudraCT forms }\end{array}$ \\
\hline
\end{tabular}

ASR: Annual Safety Report; CA: Competent authorities; CPP: Comités de Protection des Personnes; EC: Ethic Committee; EudraCT: European members database; EUREC: European Network of Research Ethics Committees; NCA: National Competent Authorities; SUSAR: Suspected unexpected serious adverse reactions

and EC would receive a summary of the ASR. This simplification of documentation is desirable not only because of EC limited resources, but also because, unlike $\mathrm{CA}$, they do not have public health inspection powers.

With a view to improving harmonisation, it would also be useful to envisage the possibility of a European authorisation for trials (centralised and/or decentralised authorisation) as for the Marketing Authorisation procedures, while retaining the sponsor's decision to opt to submit a request for trial authorisation on a national level.

Current experiences to work-share PSUR or CTFG tasks, especially setting up the Voluntary Harmonisation Procedure (VHP) are initiatives which demonstrate the growing awareness of member states and their wish to draw up a common shared assessment approach.

A centralised procedure would lead to less flexibility, more tightened timelines, possible unknown complexities, the loss of national relationship between CA and EC. This would lead to the same difficulties at each new substantial amendment.

Lastly, setting up a Community authorisation procedure for clinical trial safety would, in practice, lead to a centralised assessment by one or 2 national CA as practised within the framework of PSUR assessments. This joint assessment could apply to annual reports as well as to new safety issues, or even to all SUSAR; with regard to the latter, this would necessarily require an identical reference document for every country concerned with the trial and a definition of investigational and non-investigational drugs, applicable in an identical manner in every member country.

The proposed changes could be made within the scope of European Regulation. Once a compromise had been reached at the European level and the wording approved, the advantage would be to avoid later transposition in the regulation in each country, a source of potential discord.

However, it must be recognised that legal or regulatory difficulties could arise, which would need to be resolved beforehand. To overcome these difficulties, an approach of parliamentary representatives or European organisations such as the CTFG or the EUREC would be necessary.

\section{Conclusion}

The work carried out during the Round Table enabled fairly similar observations to emerge from various research partners on the progress of the European Directive 2001/20/CE, as well as 
desired improvements and changes to it. Beyond the necessary harmonisation between countries, the possibility of Community authorisation would require a Community Regulation. To take this further, we would need to liaise with French representatives in the European parliament or Community coordination organisations (such as the CTFG or the IWGGCP for CA, the EUREC for EC, the ECRIN to represent institutional sponsors and EFPIA for industrial sponsors). Discussion will be further enriched in 2010 by the responses made by these bodies to the recent public consultation on the Directive. ${ }^{[12]}$

As part of the attractiveness of clinical research, it would be useful to develop further performance indicators on national and European levels to help assess regulatory changes decided upon for biomedical research.

Lastly, the discussion on risk approaches adapted to each type of trial, already being debated on an academic level, was addressed in depth during the round table in an attempt to follow the diverse academic, regulatory and industrial points of view.

Participants. Michel Abiteboul (Laboratoire Quintiles), Delphine Bertram (Délégation à la Recherche Clinique, CHU, Lyon), Catherine Cornu (Ecrin, CIC, CHU, Lyon), Olivier Chassany (DRRC, AP-HP, Hôpital Saint-Louis Paris), Jacques Demotes-Mainard (Inserm, Paris), Jean-Paul Demarez (Laboratoire Pierre Fabre Médicaments), Vincent Diebolt (GipCengeps, Lyon), Claude Dubray (CIC, CHU, Clermont-Ferrand), Mats Ericson (Laboratoire Amgen), Elisabeth Frija Orvoen (Pathologies du sommeil, Hôpital Pitié-Salpêtrière, Paris), François Hirsch (Inserm, Paris), Yves Juillet (Leem, Paris), Véronique Lamarque (Laboratoire Pfizer), Rémi Le Coent (Laboratoire GlaxoSmithKline), Christian Libersa (CIC, Inserm, Hôpital Cardiologique, Lille), Christine Marey (Science Union, les Laboratoires Servier), Brigitte Marchenay (Laboratoire Roche), Armelle Mijonnet (Laboratoire Merck Sharpe \& Dohme Chibret), Valérie Plattner (Direction Recherche Clinique et Innovation, CHU, Lyon), Anne Raison (Afssaps, Saint-Denis), Patrick Rossignol (CIC, Inserm, CHU, Vandœuvre-lès-Nancy), Philippe Rusch (Faculté de Médecine, Saint-Étienne), Claire Sibenaler (Leem), Tabassome Simon (CHU Saint-Antoine, URC Est Pharmacologie, Paris).

\section{References}

1. Pehboeck D, Hohlrieder M, Wenzel V, et al. Submission of clinical studies to Ethics Committees or clinical trials registers - the authors' point of view. Intensive Care Med 2009; 35: 713-6

2. European Forum for Good Clinical Practice (EFGCP).Conference on the Impact on Clinical Research of European Legislation (FP7 program, ICREL), results § discussion, Brussels 2 Dec 2008. http: //www . efgcp. be/Downloads/confDocuments/Programme_ICREL_2_Dec_2008_ final.pdf

3. Kubiak C, De Andres-Trelles F, Kuchinke W, et al. Common definition for categories of clinical research: a prerequisite for a survey on regulatory requirements by the European Clinical Research Infrastructures Network (ECRIN).Trials 2009; 10: 95-101

4. Jardé O. Projet de Loi sur les recherches sur la personne. Assemblée Nationale, 22 janvier 2009. http: //www . assemblee-nationale. fr

5. Clinical Trials Facilitation Group: CTA assessment in member states, 22 December 2008. http://www.hma.eu/uploads/media/Assessment in_MS_public_dec_08__2_B.pdf.

6. European Network of Research Ethics Committees (EUREC). http://www eurecnet.org/index.html

7. Bertoye PH, Courcier-Duplantier S, Best N. Adaptation de la mise en oeuvre des bonnes pratiques cliniques en fonction des caractéristiques de certaines recherches. Thérapie 2006; 61: 271-7

8. Projet Optimon, OPTimisation du MONitorage : évaluation de l'efficacité et du coût de deux stratégies de monitorage pour la recherche clinique institutionnelle. Version du 4 février 2008. https://ssl2.isped. u-bordeaux 2. fr/optimon/Documents . aspx

9. Notes on good practice for research organisations in the management of a portfolio of trials 2: assessment of risk. Clinical trials tool kit issued by the Department of Health and the Medical Research Council (MRC), 2004. http://www.cttoolkit.ac.uk/_db/_documents/MPTrials2.pdf

10. Wolzt M, Druml C, Leitner D, et al. Protocols in expedited review-tackling the workload of Ethics Committees. Intensive Care Med 2009; 35: 613-5

11. Brosteanu $\mathrm{O}$, Houben $\mathrm{P}$, Ihrig $\mathrm{K}$, et al. Risk analysis and risk adapted on-site monitoring in non commercial clinical trials. Clin Trials 2009; 6: 585-96

12. European Commission. Assessment of the functioning of the Clinical Trials Directive 2001/20/EC: public consultation paperENTR/F/2/SF D (2009) 32674. 09/10/2009. http://ec.europa.eu/enterprise/sectors/ pharmaceuticals/files/clinicaltrials/docs/2009_10_09_ public-consultation-paper.pdf

Correspondence and offprints: Pascal Bilbault, Recherche Clinique Boehringer Ingelheim France, 12 rue André Huet, 51060 Reims Cedex, France.

E-mail: pascal.bilbault@boehringer-ingelheim.com 